\title{
Persistence-based temporal filtering for MODIS snow products
}

MORRISS B.F., OCHS, E., DEEB, E.J., NEWMAN, S.D., DALY, S.F., AND GAGNON, J.J.

United States Army Corps of Engineers Engineer Research and Development Center Cold Regions Research and

\section{ABSTRACT}

Single-day snow covered area (SCA) products are incomplete and often inadequate representations of ground conditions due to short term variation in cloud cover, snow cover, and sensor geometry. To mitigate these effects, we developed a by-pixel filtering algorithm to produce relatively cloud-free SCA products from 16 days of MODIS imagery. The algorithm uses previous days' data to estimate the current SCA value of each pixel and uses a simple persistence test to reduce the effects of spurious SCA/cloud classifications in the input products. To be positively

11 identified as SCA, a pixel must be snow-covered in the two most recent, cloud-free scenes of the 16-day period. We 12 applied this Time-Domain-Filtering (TDF) methodology to two single-day MODIS fractional snow cover products

13 (MOD10A1 and MODSCAG) over the MODIS period of record (2000-present) and compared the outputs to the

14 unfiltered products, to filtered maps generated using the cloud-gap-filled algorithm (CGF, Hall et al. 2010), and to

15 historical snow assessment reports from the U.S. Army Corps of Engineers Cold Regions Research and Engineering

16 Laboratory (CRREL). The CRREL reports were manually generated and quality-controlled by an analyst and are

17 treated as ground truth. We find that, when applied to MODSCAG, the TDF algorithm successfully fills in gap

18 pixels and limits the effects of snow/cloud confusion and produces a filtered product that is more consistent and

19 accurate than the MODSCAG CGF product and comparable to the MOD10A1 CGF product.

21 KEYWORDS: MODIS, snow covered area, cloud-free, gap-fill

*Correspondence to Blaine F. Morriss, United States Army Corps of Engineers Cold Regions Research and Engineering Laboratory (USACE CRREL), Hanover, NH, USA. Email: blaine.f.morriss@erdc.dren.mil. 
BACKGROUND

\section{CRREL Snow Assessments}

24 Since the winter of 2004, the Cold Regions Research and Engineering Laboratory (CRREL) of the U.S. Army

25 Engineer Research and Development Center (ERDC) has produced biweekly assessments of snow covered area 26 (SCA) and snow-water equivalent (SWE) for the major watersheds of Afghanistan using both multispectral 27 (AVHRR and MODIS) and passive microwave (SSM/I and AMSR-E) imagery (Daly et al., 2012). Accurate 28 delineation of SCA in this region is important for military transportation and operations planning as well as for 29 constraining estimates of SWE for water management and hydrological/climate modeling. The latter is particularly 30 important in areas like Afghanistan where real-time precipitation and snowpack data are scarce. Imagery from the

31 Terra Moderate Resolution Imaging Spectroradiometer (MODIS) was first used in a CRREL snow assessment in 322008 and each biweekly SCA map was generated using the most acceptable scene, based on low cloud cover and

33 proximity to the assessment day. These maps $(\mathrm{CH})$ were generated from reflectance products using unsupervised 34 classification techniques described by Daly et al., (2012) and validated qualitatively by an analyst. Due to variations 35 in cloud cover, these single day maps provided temporally inconsistent and incomplete snapshots of SCA. 36 Additionally, manually classifying and validating each scene is both time-consuming and subjective.

\section{MODIS Snow Products}

The MODIS/Terra Snow Cover Daily L3 Global 500m Grid (MOD10A1, Hall et al., 2006) is a global, gridded

39 fractional snow covered area (FSCA) product produced daily by the National Snow and Ice Data Center (NSIDC).

40 SCA is estimated using the normalized difference snow index (NDSI), which leverages the contrasting reflectance

41 of snow in the visible and shortwave infrared regions of the spectrum (Hall et al., 2002). FSCA is estimated using

42 an empirical relationship between FSCA and NDSI (Salomonson \& Appel, 2004).

43 The MODIS Snow Covered Area and Grain Size (MODSCAG) product includes gridded FSCA produced daily

44 by the Jet Propulsion Laboratory (JPL). MODSCAG uses spectral mixture analysis of the eight MODIS surface

45 reflectance bands (MOD09GA; LPDAAC, 2001) to estimate FSCA (Painter et al., 2009).

46 Both of these products are released in the MODIS sinusoidal projection and gridded to the MODIS tile system at

47 nominally $500 \mathrm{~m}(463.3 \mathrm{~m})$ pixel resolution. Examples and analysis presented in this study are limited to tile

$48 \mathrm{H} 23 \mathrm{~V} 05\left(30\right.$ to $40^{\circ} \mathrm{N}, 57.7$ to $\left.78.3^{\circ} \mathrm{E}\right)$, which encompasses the majority of Afghanistan (Figure 1A). While a 
relatively small area of interest, this region includes a wide variety of SCA environments ranging from high

50 elevation glaciated mountains to perennial lowland desert. Additionally, there is relatively little vegetation in this

51 region, so any differences in SCA detections caused by canopy will be minimized in the unfiltered products

52 allowing for better isolation of the filtering algorithms' effects.

53 The CRREL snow assessments were delivered as binary snow maps. When comparing the products to $\mathrm{CH}$, each

54 product was assumed to be binary with the FSCA $>20 \%$ set as SCA. We find this assumption appropriate for this

55 application because (1) individual pixel's FSCA retrievals from the two different detection methodologies can vary

56 widely, particularly at low FSCA, and (2) noise in MODIS band 5 tends to produce artifacts in MODSCAG's FSCA

57 estimates that can be easily ignored when considering the binary case. The FSCA threshold value was adopted

58 based on documentation for the MOD10A1 product (Riggs et al., 2006), which suggests that values below $20 \%$ are

59 often errors of commission caused by snow/cloud confusion and cloud shadows. We find such errors to be more

60 common in MODSCAG and take the 20\% FSCA threshold to be a reasonable starting point.

\section{Cloud Masking}

62 Clouds constitute the most common 'data gap' in optical imagery, though determination of which pixels are 63 cloud-covered, or for which pixels FSCA is calculated and which are masked, differs significantly by product. The

64 MODIS cloud mask (MOD35) uses a host of algorithms and 19 of MODIS's 36 bands to determine whether or not 65 MODIS is viewing clear skies (Ackerman et al., 2010). The MOD35 confidence level is an ancillary input to many 66 of the MODIS level 2 land, ocean, and atmosphere science algorithms including the MOD10- snow cover products.

67 The MOD10A1 Version 4 employed a more liberal cloud mask to mitigate overly aggressive masking and algorithm 68 confusing between clouds and snow in some regions; however, Version 5 does not use the liberal mask in an effort 69 to provide a more globally consistent product but may over-classify cloud cover as a result (Riggs et al., 2003; Hall 70 et al., 2006; Riggs \& Hall, 2007). In an effort to avoid overly aggressive cloud cover classification, MODSCAG 71 uses a cloud mask based on surface reflectance from the MOD09GA, modified by grain size estimates of snow and 72 cloud. Clouds with relatively large particle size are mapped as snow, and, similarly, snow with too-fine particle 73 sizes are mapped as clouds (Painter et al., 2009). This results in lower cloud cover estimates when compared with 74 the MOD10A1 and potentially more accurate characterization of FSCA (Rittger et al., 2013) but also more false 75 positives in SCA detections. In each case, we assume that all calculated values of FSCA $(0-100 \%)$ are valid 76 inputs and that all other values, whether cloud, water, no decision, or the variety of other negative flags present in 
77 the products, are treated as 'cloud' and will be attempted to be filled by the algorithm. For simplicity, further 78 discussion of 'cloudy' pixels will pertain to any pixel that does not have an FSCA/SCA (whether binary or 0 -

$79100 \%$ ) value. False positives due to snow/cloud confusion are the largest source of error in the input imagery in

80 terms of FSCA/SCA, which, when coupled with significant differences in cloud covered area extent in the two input

81 products, provides a compelling test environment for the algorithm.

\section{Cloud Mitigation and Removal}

83 Gap-filling methods use the values of temporally or spatially adjacent pixels to interpolate the values of pixels in 84 data gaps which may be caused by clouds, sensor or illumination geometry, or sensor malfunction. Spatial gap85 filling such as kriging use data from surrounding non-gap pixels to estimate the values of missing data (Rossi et al.,

86 2004). Temporal filtering leverages non-gap values of a pixel from nearby scenes within a time series to estimate 87 the value in the gap, often using curve-fitting on non-gap data from both before and after the gap (Jönsson \& 88 Eklundh, 2004). Spatio-temporal gap-filling methods use a combination, usually in alternating steps, of spatial and 89 temporal techniques to incrementally remove data gaps.

90 Several cloud removal strategies have been developed specifically for MODIS snow products using a variety of 91 spatial and temporal filtering techniques. The temporal and spatial variability in snow cover makes spatial filters 92 somewhat unreliable; however, Gafurov and Bárdossy (2009) used one in conjunction with elevation as part of a 93 multi-step spatio-temporal algorithm, assigning snow cover to gap pixels that were both higher elevation and 94 adjacent to snow-covered pixels. Temporal filters are powerful because cloud cover is more variable than snow 95 cover. MODIS provides the unique advantage of collecting both a morning and afternoon pass each day from 96 NASA Terra and Aqua satellites, respectively. This time difference alone can remove significant clouds from an 97 aggregated daily product (Parajka \& Blöschl, 2008); however, we limited imagery used in this study to Terra both to 98 illustrate the filtering algorithm's applicability to other data sets that do not have twice-daily acquisitions and 99 because Aqua MODSCAG was not available for analysis. Multi-day filtering solutions use time windows of 100 different sizes to either fill gap data with recently available data (Hall et al., 2010) or interpolate gap data using 101 curve-fitting. Spatio-temporal interpolation and smoothing techniques, like that developed by Dozier and others 102 (2008), provide powerful cloud-removal but are computationally expensive and can retain and exacerbate spurious 103 SCA detections from the input imagery. 
104 Our goal was to create an efficient gap-filling solution that required only daily FSCA/SCA as an input and that 105 could be applied to methodologically different input sources while producing consistent, commensurate results.

106 This approach provides flexibility in terms of data availability and portability with regards to location, data source,

107 and overall processing scheme. Both the need for and application of our process are shown in Figure 2, which

108 compares data from MODSCAG and MOD10A1 that was filtered using our time-domain-filtering (TDF) algorithm

109 and the cloud-gap-filled (CGF) algorithm (Hall et al., 2010). Fundamentally, the CGF algorithm outputs the most

110 recent cloud-free value (SCA or no SCA) for each pixel. In the absence of temporal or spatial curve-fitting solutions

111 that can extrapolate SCA extent into areas with no cloud-free data, using the last clear view as a proxy for unknown

112 data works well as long as the input data is reliably accurate. For the MOD10A1, which is relatively conservative in

113 its SCA detections (and aggressive in its cloud masking), this works well; however, applying the CGF algorithm to

114 MODSCAG resulted in highly variable, high magnitude SCA extent due to errors of commission in the daily SCA

115 input. The TDF filtering algorithm aims to reconcile the differences in the input imagery and produce more or less

116 equivalent and accurate estimates of SCA.

\section{METHODS}

\section{Temporal Filtering}

119 Between November 1 and June 1 for each year of the period of record (2000-2014), the chance of cloud cover or 120 invalid data in any pixel in H23V05 in either MOD10A1 or MODSCAG was $12.7 \%$; however, some pixels were 121 cloudy up to $48.7 \%$ of the time (Figure $3 \mathrm{~A}$ ). We note that the spatial distribution of mean cloud presence is similar 122 to that of mean snow presence (Figure 1B), suggesting strong orographic effects, particularly in the Hindu Kush. 123 Despite clouds being common, we find mean cloud persistence, or the average time between cloud-free scenes, to be 124 less than a day (Figure 3B) at Terra MODIS' temporal scale (1 day). This cloud cover transience gives us the 125 opportunity to fill in gaps in the current day's imagery with contemporary data. Because we use multiple days' 126 imagery as inputs and assume that cloud cover is more variable than snow cover, we choose to limit our outputs to 127 SCA that is persistent on a multi-day scale. Each output pixel's value is based on up to 16 daily images (Figure 4, 128 panels 0-15), though only the two most recent cloud-free images influence the value of the outputs. This approach 129 limits errors of commission, false positives, in snow cover that arise from cloud and cloud shadow misclassification 130 in both the MOD10A1 and MODSCAG detection algorithms; this phenomenon is readily visible on the edges of the 
131 cloud in the northwest of Figure 4A. If SCA is present in both of the two most recent cloud-free images, the output 132 pixel's value (Figure 4C) is set to the FSCA value of the most recent image. If either pixel's value in the cloud-free 133 pair is snow-free, the output pixel is assumed to be snow-free. This requirement sometimes filters transient SCA

134 from the output product in addition to spurious SCA but creates a conservative and robust delineation of SCA 135 extent. In the rare occurrence that a pixel does not have two cloud-free readings in the 16 input scenes, the output is 136 cloud. In cases where there is only one cloud-free value during the 16 day period, we retain that pixel's value in the 137 output, ignoring the persistence requirement. We define a decision age (Figure 4B) equal to the number of days 138 elapsed between 'today' (day 0 in Figure 4A,0) and the oldest image that contributes to the output.

\section{Masking}

140 Spurious classification of clouds, water bodies, and land features (salt flats) as SCA, as determined by visual 141 inspection of the daily MODIS surface reflectance product (MOD09GA), is common in both MODSCAG and 142 MOD10A1. To mitigate the effects of these misclassifications, pixels with FSCA values less than $20 \%$ were 143 ignored (Riggs et al., 2006; Hall et al., 2010). Additionally, a 5-year historical water mask was created using visible 144 and near infrared bands of the MOD09GA from November to April, 2008-2013. Rivers were extrapolated from the 145 USGS HydroSHEDS flow accumulation data set (Lehner et al., 2008), resampled to $500 \mathrm{~m}$ resolution. These pixels 146 were assumed to be snow-free in all outputs (and cloud-free when assessing data age and cloud persistence). A 147 mean historical snow mask was created using the MOD10A2 product, the 8-day maximum SCA extent of the daily 148 MOD10A1, over the period of record. Those regions where snow did not appear in more than 5\% of winter, cloud149 free, unfiltered scenes were assumed to be snow-free in all outputs. Because the maximum extent map 150 overestimates daily SCA, this final exclusion has little effect on the final product, though it can produce overly 151 conservative estimates during anomalously large snow events. It was employed to further limit the effects of water 152 bodies and other persistent land features that were commonly misclassified as snow in this region but may not be as 153 positively impactful in other locales.

\section{Validation}

155 When comparing total SCA between products we use the mean difference, median difference, and root mean 156 squared error (RMSE). These summary statistics are limited to the winter season (Nov 1 to June 1) for the period of 
157 record to avoid anomalously low errors associated with the persistent summer snowfields and glaciers in the region.

158 The $\mathrm{CH}$ maps also fall within this period. RMSE is defined as:

$$
R M S E=\sqrt{\frac{1}{N} \sum_{t=1}^{N}\left(S C A_{A, t}-S C A_{B, t}\right)^{2}}
$$

$\mathrm{N}$ is the total number of days in the compared time series $\left(S C A_{A}\right.$ and $\left.S C A_{B}\right)$, while $t$ is the ordinal day. For those comparisons involving the CRREL reports, $\mathrm{N}=110$ and $\mathrm{t}=1$ corresponds to 25 November 2006.

163 While total snow covered area is perhaps the most commonly derived parameter from SCA maps, we also 164 compared the different products to $\mathrm{CH}$ spatially. To assess this, we track three possible outcomes for each pixel in those products compared to $\mathrm{CH}$ : true positives $(T P)$, false positives $(F P)$, and false negatives $(F N)$. Using these, we calculate the following performance statistics:

$$
\text { Precision }=\frac{T P}{T P+F P}
$$

$$
F=2 \frac{\text { Precision } \times \text { Recall }}{\text { Precision }+ \text { Recall }}=\frac{2 T P}{2 T P+F P+F N}
$$

170 Precision is the chance that a pixel identified as snow has snow, while Recall is the probability of identifying a

171 snow-covered pixel. $F$ score is a combined statistic that describes the overall accuracy of the classification but

172 ignores true negatives, effectively considering only those pixels that were snow-covered in one of the products being 173 compared. This omission is important for assessing a phenomenon as spatially and temporally variable as SCA 174 because it prevents snow-free areas from inflating the perceived accuracy (Rittger et al., 2013). For example, a 175 scene with 5\% snow cover that was classified as totally snow-free would be $95 \%$ accurate but have Precision, 176 Recall, and $F$ score of $0 \%$. The same scene with half of the snow correctly classified would be $97.5 \%$ accurate but 177 have an $F$ score of $67 \%$. This provides a sensitive accuracy assessment that is not biased by either the extent of the 178 assessment area or seasonal changes in SCA.

\section{RESULTS}

180 Daily TDF and CGF SCA maps were produced using imagery from February 17, 2000 to December 31, 2014. 181 Figure 4 shows example inputs and outputs for MODSCAG TDF from 5 March 2003. Panel A shows the unfiltered 182 MODSCAG on that day, which includes several areas of low-FSCA that are probably erroneous, as determined by 
183 inspection of the surface reflectance data and the MOD10A1. The 16 input images used by TDF are shown in the 184 upper right panel, and the filtered image appears in panel C, notably with the low-FSCA pixels in the northwest 185 removed and the cloudy pixels removed.

186 Mean cloud cover in filtered images was 1,675 pixels $\left(360 \mathrm{~km}^{2}\right)$ for MODSCAG and 26,791 pixels $\left(5,751 \mathrm{~km}^{2}\right)$ 187 for MOD10A1 or $0.03 \%$ and $0.5 \%$ of the tile, respectively. We chose to retain some cloud-covered pixels rather 188 than extend the filtering window both out of concerns for the age of the data in the final product as well as the 189 processing time; however, dynamically updating the filtering window on a per pixel basis to create a completely 190 cloud-free product does not add significant processing time after creating the first product in a series because each 191 day's product can be created from the previous day's filtered SCA, the corresponding age rasters, and the new 192 unfiltered input. For all images and pixels in the period of record, mean decision age was 1.5 and 2.4 days for 193 MODSCAG and MOD10A1, respectively. The daily mean decision age was highly variable but generally greatest 194 in January and February (Figure 2D). With the exception of brief periods with missing imagery, when we assumed 195 all pixel's values to be cloud, daily mean decision age for MODSCAG was less than a week. Mean decision ages 196 for those pixels determined to be SCA were expectedly higher than the mean, about a day longer for both inputs, and 197 relatively uniform, spatially (Figure 3C).

198 When evaluating different estimates of total SCA, we started by comparing products from different inputs that had 199 been subject to the same filtering process. MODSCAG and MOD10A1 are derived from the same sensor data, 200 though they use different techniques to estimate SCA. It is then reasonable to expect that the filtered products 201 would observe a similar SCA signal. While comparison of the filtered products to $\mathrm{CH}$ allows us to assess the 202 accuracy of the filtered products, this comparison is limited to 110 scenes. Inter-comparison of total SCA between 203 products allows us to extend the accuracy assessment by proxy; if we then identify which filtered product is most 204 similar to $\mathrm{CH}$, we can draw conclusions about how that product compares to the others over a much larger number 205 of scenes. While the unfiltered products are not ultimately of interest in this study, it is notable how different they 206 are $\left(\right.$ RMSE $\left.=102.2 \times 10^{3} \mathrm{~km}^{2}\right)$ from each other. The majority of that difference (mean difference of $92.6 \times 10^{3} \mathrm{~km}^{2}$ 207 more SCA in MODSCAG) is due to many fewer clouds in MODSCAG than in MOD10A1 (mean difference = $208121.3 \times 10^{3} \mathrm{~km}^{2}$ ). Filling in the clouds using CGF alleviates some of these differences, reducing RMSE to $72.3 \mathrm{x}$ $20910^{3} \mathrm{~km}^{2}$; TDF further improves the agreement $\left(\operatorname{RMSE}=41.2 \times 10^{3} \mathrm{~km}^{2}\right)$. Because TDF requires SCA persistence 210 and neither CGF nor TDF has a mechanism to add SCA outside of gap pixels, it follows that increased agreements 
211 in total SCA estimates can be largely attributed to reducing SCA detections in MODSCAG. The TDF persistence 212 test also removes SCA from MOD10A1, which, due to its more aggressive cloud mask, results in anomalously low 213 total SCA estimates during cloudy periods and likely underestimates of SCA in general. Under the assumption that 214 the MOD10A1 CGF (for which CGF was developed and validated by Hall et al., 2010) is accurate, we compared the 215 MODSCAG TDF to the MOD10A1 CGF, and found that they are in better agreement than the TDF products are to 216 each other (RMSE $=17.9 \times 10^{3} \mathrm{~km}^{2}$ ). While this study focused on binary SCA because of the limitations of $\mathrm{CH}$ and 217 the wide use and applicability of binary SCA products, we did consider the fractional case in the inter-product 218 comparisons of total SCA. In most cases, errors were reduced when using FSCA as algorithm inputs; this is 219 particularly notable in the TDF comparison. In general FSCA comparisons, MOD10A1 tends to detect fewer SCA 220 pixels with an average higher FSCA than MODSCAG. Coincidental balancing of these tradeoffs results in lower 221 errors when comparing total SCA, but this is not supported by the spatial statistics. Statistics for select comparisons 222 of total SCA, including those above, appear in Table 1.

223 Comparing the unfiltered, CGF, and TDF results to $\mathrm{CH}$ tell a similar story. As we assumed above, MOD10A1 224 CGF is a very accurate measure of total SCA (RMSE $\left.=26.3 \times 10^{3} \mathrm{~km}^{2}\right)$ as compared to $\mathrm{CH}$. Again, we see poorer 225 performance of TDF when applied to the MOD10A1, resulting in lower accuracy $\left(28.5 \times 10^{3} \mathrm{~km}^{2}\right)$ than the 226 MOD10A1 CGF, and a reversal in sign of the mean difference $\left(-7.4 \times 10^{3} \mathrm{~km}^{2}\right)$ due to removal of SCA by the 227 persistence check, as previously suggested; however, we see dramatic improvements in MODSCAG TDF over 228 MODSCAG CGF and, ultimately, a similar RMSE $\left(29.5 \times 10^{3} \mathrm{~km}^{2}\right)$ to the MOD10A1 CGF. Summary statistics 229 appear in Table 1. For all methods except MOD10A1 TDF, error tends to manifest in overestimation of SCA 230 relative to $\mathrm{CH}$ (Figure 5). Among the tested data sets, MOD10A1 CGF and MODSCAG TDF minimize this error 231 and exhibit the least variance.

232 Analysis of the spatial accuracy of the filtered products supports the previous findings. The most accurate, with 233 an $F$ score of 0.906 , was the MOD10A1 CGF, while MODSCAG TDF $(\mathrm{F}=0.894)$ was an improvement over 234 MODSCAG CGF $(\mathrm{F}=0.877)$, and MOD10A1 TDF $(\mathrm{F}=0.884)$ was less accurate than its counterpart. The 235 tendency of MODSCAG to overestimate SCA is evidenced in the high Recall, which is driven by false negatives, of 236 both filtered products; it is then no surprise that the improvement in Precision between MODSCAG TDF and CGF 237 was over 5\%. Again, MOD10A1 TDF shows persistent underestimation as evidenced by a 7\% drop in recall as 238 compared to MOD10A1 CGF. Spatial accuracy statistics appear in Table 2. 


\section{DISCUSSION}

240 Our results show that, when applied to unfiltered MODSCAG imagery, the persistence-based time-domain-

241 filtering (TDF) produces a more consistent and accurate gap-filled SCA map than the cloud-gap-filled (CGF)

242 methodology. CGF, which retains the value of the last cloud-free pixel as the output, causes false positive

243 misclassifications of SCA, commonly found adjacent to clouds in MODSCAG, to appear in the filtered product.

244 TDF prevents many of these misclassifications by requiring consecutive (considering only cloud-free detections)

245 snow-covered readings to produce a snow pixel in the filtered output. While MODSCAG TDF and MOD10A1 CGF

246 minimized error in total SCA estimates when compared to $\mathrm{CH}$, they also performed best spatially. As both

247 MODSCAG and MOD10A1 detections methodologies differ from that used to generate $\mathrm{CH}$, some differences are

248 expected. Figure 6 maps the spatial accuracy statistics across the comparison region for the filtered MODSCAG

249 products and MOD10A1 CGF as color composites where the Red, Green, and Blue channels are mean Recall, mean

250 F score, and mean Precision, respectively. Each color channel's output is scaled to the dynamic range of its

251 variable, so the color combinations are indicative of the relative contribution of each variable rather than their

252 absolute magnitudes; this highlights spatial trends in the accuracy statistics and, when considered in combination

253 with their scene-wide summary statistics found in Table 2, paints a more complete picture of how each algorithm

254 performs. MODSCAG CGF displays large regions of relatively low Precision and high Recall, consistent with

255 common false positives. These red regions are largely absent in the MODSCAG TDF and MOD10A1 CGF panels,

256 replaced by more nuanced regional inaccuracies. These two panels show very similar spatially distributed error,

257 largely confined to low-elevation regions where snow is less common. These regions show a more mottled

258 appearance than in the top panel because they are not dominated by a particular error type and are mapped over

259 smaller dynamic ranges due to their higher overall accuracy. The MODSCAG TDF map as a whole, when

260 compared to the MOD10A1 CGF, has a slightly bluer hue. This suggests that TDF's logic is systematically

261 removing some true positive snow pixels, resulting in relatively lower Recall scene-wide, a problem which was

262 immediately noticeable in the MOD10A1 TDF; however, the improvements in both spatial accuracy and total SCA

263 estimation far outweigh this bias. 


\section{CONCLUSION}

265 Clouds, sensor and solar geometry, and sensor malfunction introduce gaps into multispectral remotely sensed

266 data. In many cases, these data gaps are temporally variable, and a time series of imagery can be used to estimate

267 the values of gap pixels. We developed an algorithm to remove clouds and limit false positives due to cloud

268 confusion from daily snow covered area products. The value of cloudy pixels was assumed to be snow-covered if

269 the two most recent cloud-free days were snow-covered. Because cloud cover is generally more variable than SCA

270 on a daily time scale, this simple persistence test limits false positives in SCA caused by cloud/snow confusion that

271 are common in daily products. While we use up to 16 days of imagery to estimate SCA for any given day, we find

272 that most gaps are filled in just a few days despite the persistence test. We found that, when compared to manually

273 derived historical SCA estimates (CH), the Time-Domain-Filtered (TDF) MODSCAG and Cloud-Gap-Filled (CGF)

274 MOD10A1 produced the most consistent and accurate estimates of SCA. MODSCAG CGF and MOD10A1 TDF

275 tended to overestimate and underestimate SCA, respectively, and exhibited more classification inaccuracies

276 spatially. While the most similar to the CH, MODSCAG TDF and MOD10A1 CGF were also the most similar to

277 each other of those tested. This reconciliation of SCA signals is important because the inputs products, while using

278 different SCA detection methodologies, are derived from the same sensor data and should arrive at similar

279 estimations of SCA in a filtered product. This method for removing spurious SCA detections from MODSCAG,

280 while interpolating the values of cloud-covered pixels, results in a product that is more comparable, spatially, to

281 existing filtered SCA data sets and allows the user to potentially leverage MODSCAG's fractional snow cover

282 capabilities without the burden of the extra snow/cloud-confused SCA misclassifications.

\section{ACKNOWLEDGEMENTS}

284 This work was supported through funding from the U.S. Army Corps of Engineers Reachback Operation Center

285 (UROC) and the Cold Regions Research and Engineering Laboratory. The MOD10A1 data product is courtesy of

286 the National Snow and Ice Data Center (NSIDC) Distributed Active Archive Center (DAAC), NSIDC, Boulder,

287 Colorado. The MOD09GA data product is courtesy of the online Data Pool at the NASA Land Processes (LP)

288 DAAC, USGS/Earth Resources Observation and Science (EROS) Center, Sioux Falls, South Dakota. Both the

289 MOD09GA and MOD10A1 can be accessed free-of-charge via NASA Earth Observing System Data and 
290 Information System (EOSDIS) Reverb (http://reverb.echo.nasa.gov/reverb/). MODSCAG data product is courtesy

291 of the NASA Jet Propulsion Laboratory (JPL), Pasadena, California.

292

293

294

295

296

297

\section{REFERENCES}

Ackerman S, Frey R, Strabala K, Lui Y, Gumley L, Baum B, Menzel P, 2010. Discriminating clear-sky from cloud with MODIS algorithm theoretical basis document (MOD35), Version 6.1. ATBD Reference Number: ATBDMOD-06.

Daly SF, Vuyovich CM, Deeb EJ, Newman SD, Baldwin TB, Gagnon JJ, 2012. Assessment of the snow conditions in the major watersheds of Afghanistan using multispectral and passive microwave remote sensing. Hydrol Process 26: 2631-2642.

Dozier J, Painter TH, Rittger K, Frew J, 2008. Time-space continuity of daily maps of fractional snow cover and albedo from MODIS. Adv Wat Res 31: 1515-1526. http://dx.doi.org/10.1016/j.advwatres.2008.08.011.

Gafurov A, Bárdossy A, 2009. Cloud removal methodology from MODIS snow cover product. Hydrol Earth Syst Sci 13:1361-1373. http://dx.doi.org/10.5194/hess-13-1361-2009.

Hall DK, Riggs GA, 2007. Accuracy assessment of the MODIS snow products, Hydrol Process 21(12):1534-1547. http://dx.doi.org/10.1002/hyp.6715.

Hall DK, Riggs GA, Foster JL, Sujay VK, 2010. Development and evaluation of a cloud-gap-filled MODIS daily snow-cover product. Remote Sens Environ 114:496-503.

Hall DK, Riggs GA, Salomonson VV, DiGirolamo NE, Bayr KJ, 2002. MODIS snow-cover products. Remote Sens Environ 83:181-94. http://dx.doi.org/10.1016/S0034-4257(02)00095-0.

Hall DK, Riggs GA, Salomonson VV, 2006. MODIS/Terra Snow Cover Daily L3 Global 500m Grid V005 (MOD10A1), March, 2000-June, 2014. Boulder, Colorado USA: National Snow and Ice Data Center. Digital media.

Jönsson P, Eklundh L, 2004. TIMESAT - a program for analyzing time-series of satellite sensor data. Comput Geosci 30: 833-845. http://dx.doi.org/10.1016/j.cageo.2004.05.006.

Lehner B, Verdin K, Jarvis A, 2008. New global hydrography derived from spaceborne elevation data. Eos, Trans Amer Geophys Union 89(10): 93-94. 
NASA Land Processes Distributed Active Archive Center (LP DAAC), 2001. Surface Reflectance Daily L2G Global $1 \mathrm{~km}$ and 500m (MOD09GA). USGS/Earth Resources Observation and Science (EROS) Center, Sioux Falls, South Dakota.

Painter TH, Rittger K, McKenzie C, Slaughter P, Davis RE, Dozier J, 2009. Retrieval of subpixel snow covered area, grain size, and albedo from MODIS. Remote Sens Environ 113:868-79. http://dx.doi.org/10.1016/j.rse.2009.01.001.

Parajka J, Blöschl G, 2008. Spatio-temporal combination of MODIS images - potential for snow cover mapping. Water Resour Res 44(3): W03406. http://dx.doi.org/10.1029/2007WR006204.

Salomonson VV, Appel I, 2004. Estimating fractional snow cover from MODIS using the normalized difference snow index. Remote Sens Environ 89:351-60. http://dx.doi.org/10.1016/j.rse.2003.10.016.

Riggs GA, Hall DK, Salomonson VV, 2006. MODIS Snow Products User Guide to Collection 5. http://modis-snowice.gsfc.nasa.gov/uploads/sug_c5.pdf.

Rittger K, Painter TH, Dozier J, 2013. Assessment of methods for mapping snow cover from MODIS. Adv Wat Res 51:367-380. http://dx.doi.org/10.1016/j.advwatres.2012.03.002.Rossi RE, Dungan JL, Beck LR, 1994. Kriging in

\section{FIGURE CAPTIONS}

334 Figure 1. (A) Map of geopolitical boundaries, watersheds (red), and elevation for Afghanistan. Black hashed line

335 denotes extent of MODIS tile H23V05. (B) SCA presence or the chance of an area being snow covered between 336 November and June (2000-2014), derived from daily MODSCAG and MOD10A1 (n=6068). Areas with no SCA 337 detections during the period of record (POR) appear grey.

338 Figure 2. Filtered SCA Time Series for Select Years. This figure shows two of the cloudier years in the period of 339 record to highlight both the need and application of the TDF algorithm. When applied to MODSCAG, the cloud340 gap-filled (CGF) logic results in a noisy, high magnitude SCA signal (light red) that is not consistent with either the 341 other products nor the CRREL historic (CH) maps. Similarly, MOD10A1 TDF (blue) tends to underestimate total 
342 SCA relative to the other products, including major failures during very cloudy periods (March 2003) due to its 343 aggressive cloud mask. The black and grey lines show the area covered by clouds for 16 days straight, prior to and

344 including that day, on any given day for MODSCAG and MOD10A1, respectively. MOD10A1 CGF (light blue) and

345 MODSCAG TDF (red) were found to perform best both when compared to the other filtered data sets as a data

346 reconciliation exercise and when compared to the $\mathrm{CH}$ data set. X-axis spans 1 October, 2002, to 30 September 2004

347 with ticks on the first of each year.

348 Figure 3. Cloud Presence and Persistence. The first panel (A) shows the chance of cloud cover in the tile (in either 349 unfiltered product) between November and June in each year of the POR. Despite significant cloud cover,

350 particularly in the higher elevations, the average number of days between the current day and a cloud-free scene (B)

351 is short. Panel $\mathbf{C}$ shows the mean decision age, or the age of the oldest data that appears in the filtered SCA map for 352 all snow-covered pixels, most often the age of the older image in the cloud-free pair. Mean decision age across the 353 entire tile, calculated on each day of the POR, is plotted in panel D for MODSCAG (red) and MOD10A1 (blue)

354 TDF. These differ considerably because of the different cloud layers in the unfiltered products.

355 Figure 4. Example algorithm inputs, temporal diagnostic, and output from March 5, 2003. MODSCAG from this 356 date $(\mathbf{A}, \mathbf{0})$ shows significant cloud cover (red). Our algorithm uses up to 15 previous days' data (numbered panels, 357 increasing backwards in time) to estimate current SCA (C). The decision age (B) for this day, or the time since a 358 pixel recorded two cloud-free readings, illustrates the effective age of each of the output pixels and constrains the 359 temporal resolution and accuracy of the product.

360 Figure 5. Comparison of Filtered SCA Products and CRREL Historic (CH) Maps. This scatterplot details 361 individual comparisons between the filtered maps and $\mathrm{CH}$. The two data sets that performed best, MODSCAG TDF 362 and MOD10A1 CGF, appear in bright red and blue, respectively. Summary statistics for these comparisons appear 363 in Table 1.

364 Figure 6. Spatial Distribution of Accuracy Statistics. These three panels show the spatial distribution of the 365 accuracy statistics as color composite images where R,G,B = mean Recall, mean F score, mean Precision for both 366 filtered MODSCAG products and MOD10A1 CGF. Each band is normalized to its own dynamic range to maximize 367 contrast and, therefore, represents the relative distribution of the error signal, as opposed to its absolute magnitude, 
368 which is summarized in Table 2. This means that areas dominated whose error is dominated by false positives

369 appear in red and those dominated by false negatives appear in blue. Brightness differences depend on the relative

370 contributions of the three variables and are dominated by Recall and Precision (there are no green areas as it is

371 impossible to have a high $F$ score with low Precision and Recall). The large bright red regions in the upper panel

372 denote areas of high Recall, low F score, and low Precision in the MODSCAG CGF. This corresponds to areas that

373 were often classified as snow (this incidentally results in high Recall) but were often false positives. The duller reds

374 in the interior of the upper panel highlight areas that were more accurate overall (uniformly greater $F$ score and

375 Precision) but still showed more false positives (greater Recall than $F$ score or Precision). 
Table 1.

Summary statistics of estimates of total SCA for winters over the period of record

\begin{tabular}{|c|c|c|c|}
\hline \multirow[b]{2}{*}{ Comparison } & \multirow[b]{2}{*}{ Statistic } & \multicolumn{2}{|c|}{ Area $\left(1000 \mathrm{~km}^{2}\right)$} \\
\hline & & Binary & Fractional \\
\hline \multirow[t]{3}{*}{ MODSCAG | MOD10A1 Unfiltered } & RMSE $^{1}$ & 102.2 & 61.3 \\
\hline & Mean difference ${ }^{2}$ & 92.6 & 52.6 \\
\hline & Median difference & 85.8 & 47.1 \\
\hline \multirow[t]{3}{*}{ MODSCAG | MOD10A1 Unfiltered (Cloud) } & RMSE & 138.6 & \\
\hline & Mean difference & -121.3 & \\
\hline & Median difference & -115.8 & \\
\hline \multirow[t]{3}{*}{ MODSCAG | MOD10A1 CGF } & RMSE & 72.3 & 28.5 \\
\hline & Mean difference & 60.7 & 6.5 \\
\hline & Median difference & 53.9 & 3.2 \\
\hline \multirow[t]{3}{*}{ MODSCAG | MOD10A1 TDF } & RMSE & 41.2 & 22.0 \\
\hline & Mean difference & 35.4 & 0.4 \\
\hline & Median difference & 31.1 & -1.4 \\
\hline \multirow[t]{5}{*}{ MODSCAG TDF | MOD10A1 CGF } & RMSE & 17.9 & 25.2 \\
\hline & Mean difference & 10.8 & -17.7 \\
\hline & Median difference & 9.6 & -14.6 \\
\hline & Mean SCA extent & 254.2 & \\
\hline & MODIS Tile & 1236.4 & \\
\hline \multirow[b]{2}{*}{ Comparison } & & \multicolumn{2}{|c|}{ Area $\left(1000 \mathrm{~km}^{2}\right)$} \\
\hline & Statistic & MODSCAG & MOD10A1 \\
\hline \multirow[t]{3}{*}{ Unfiltered $\mid \mathrm{CH}$} & RMSE & 37.4 & 74.7 \\
\hline & Mean difference & 13.7 & -53.9 \\
\hline & Median difference & 17.3 & -42.2 \\
\hline \multirow[t]{3}{*}{$\mathrm{CGF} \mid \mathrm{CH}$} & RMSE & 45.6 & 26.3 \\
\hline & Mean difference & 39.2 & 11.2 \\
\hline & Median difference & 34.1 & 10.9 \\
\hline \multirow[t]{5}{*}{$\mathrm{TDF} \mid \mathrm{CH}$} & RMSE & 29.5 & 28.5 \\
\hline & Mean difference & 19.5 & -7.4 \\
\hline & Median difference & 17.6 & -5.5 \\
\hline & Mean SCA extent & 200.9 & \\
\hline & $\mathrm{CH}_{\mathrm{Mask}}^{3}$ & 661.1 & \\
\hline
\end{tabular}

${ }^{1}$ RMSE is defined in Eq. (1).

${ }^{2}$ Difference is total SCA (or cloud) in the first product listed in the comparison minus the second.

${ }^{3}$ Area of interest monitored by $\mathrm{CH}$ varied over time. Comparisons with $\mathrm{CH}$ are limited to the area intersected by the 110 maps. 
Table 2.

By-pixel comparison of filtered products to CRREL historic maps

\begin{tabular}{c|ccc}
\hline & Precision & Recall & F score \\
\hline MOD10A1 CGF & .879 & .935 & .906 \\
MODSCAG CGF & .797 & .975 & .877 \\
MOD10A1 TDF & .903 & .865 & .884 \\
MODSCAG TDF & .849 & .943 & .894 \\
\hline
\end{tabular}


Figure 1
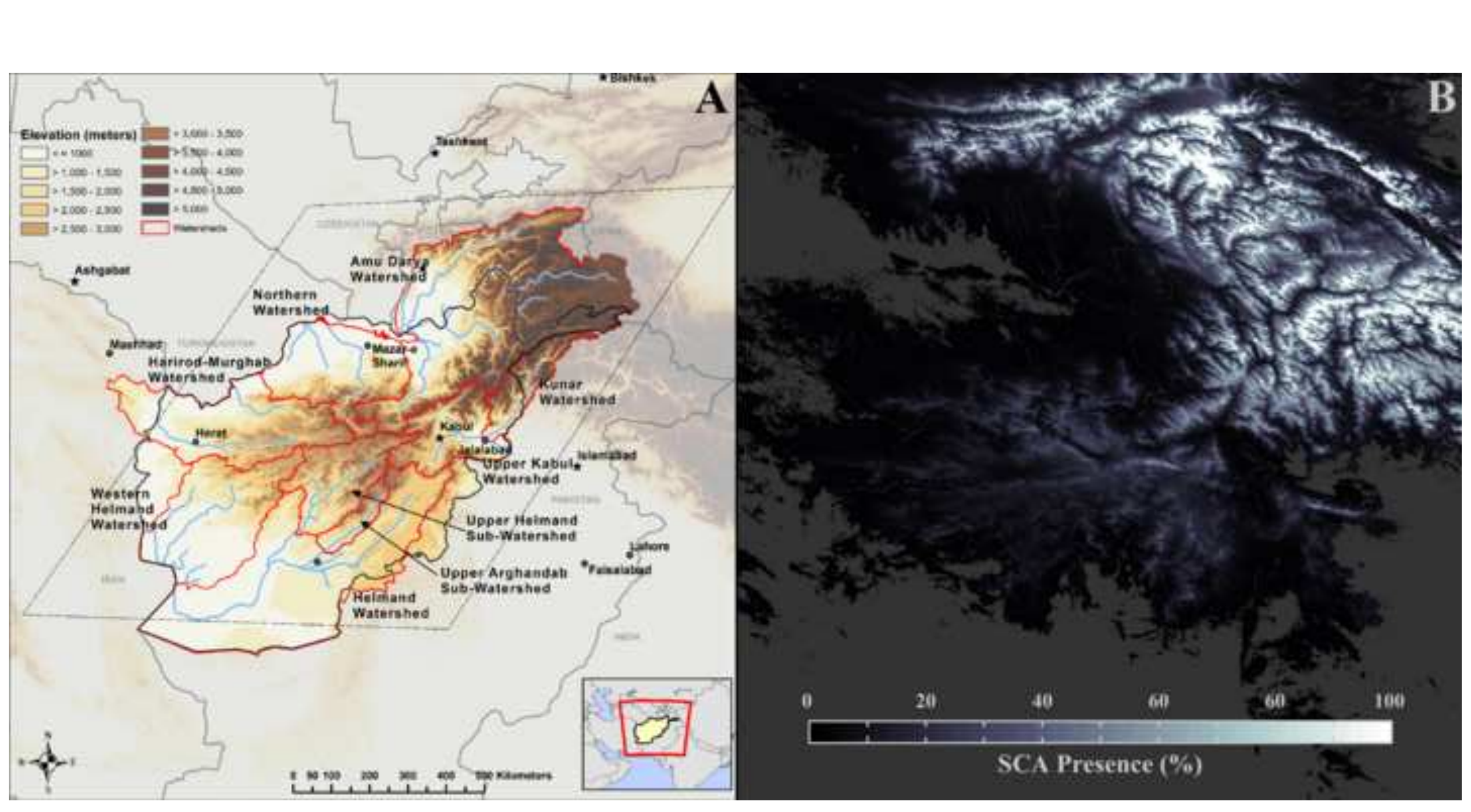


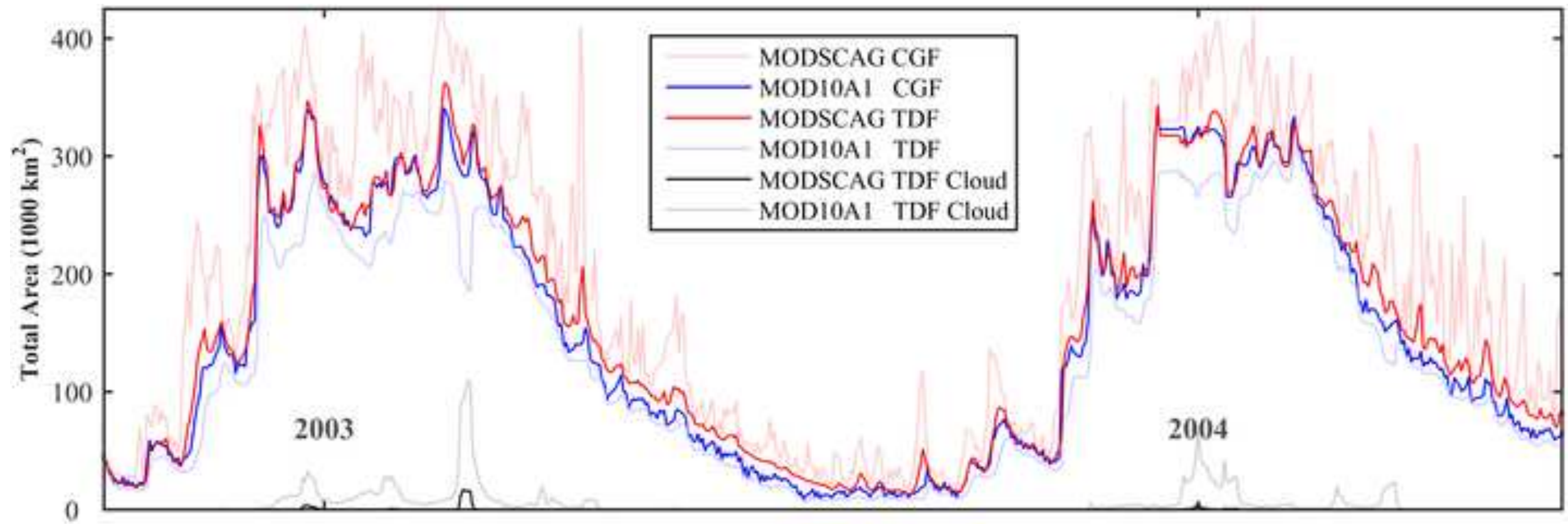




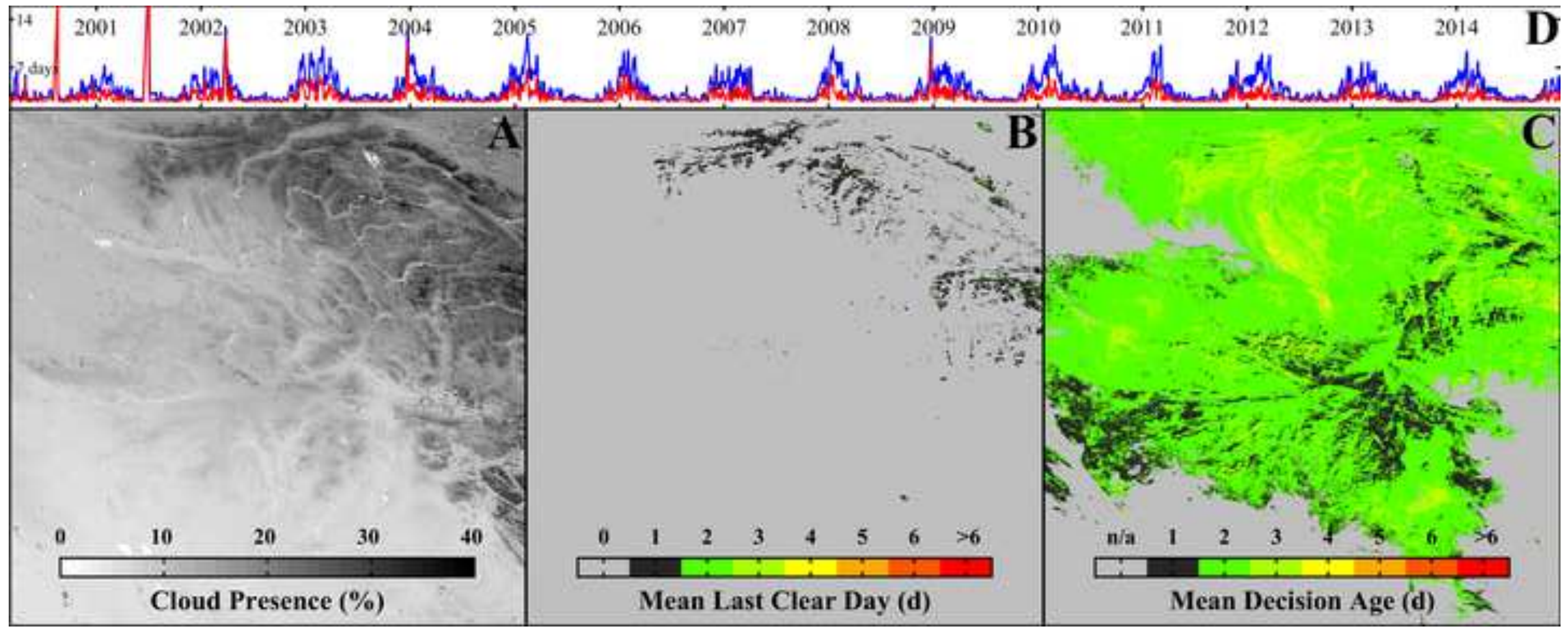




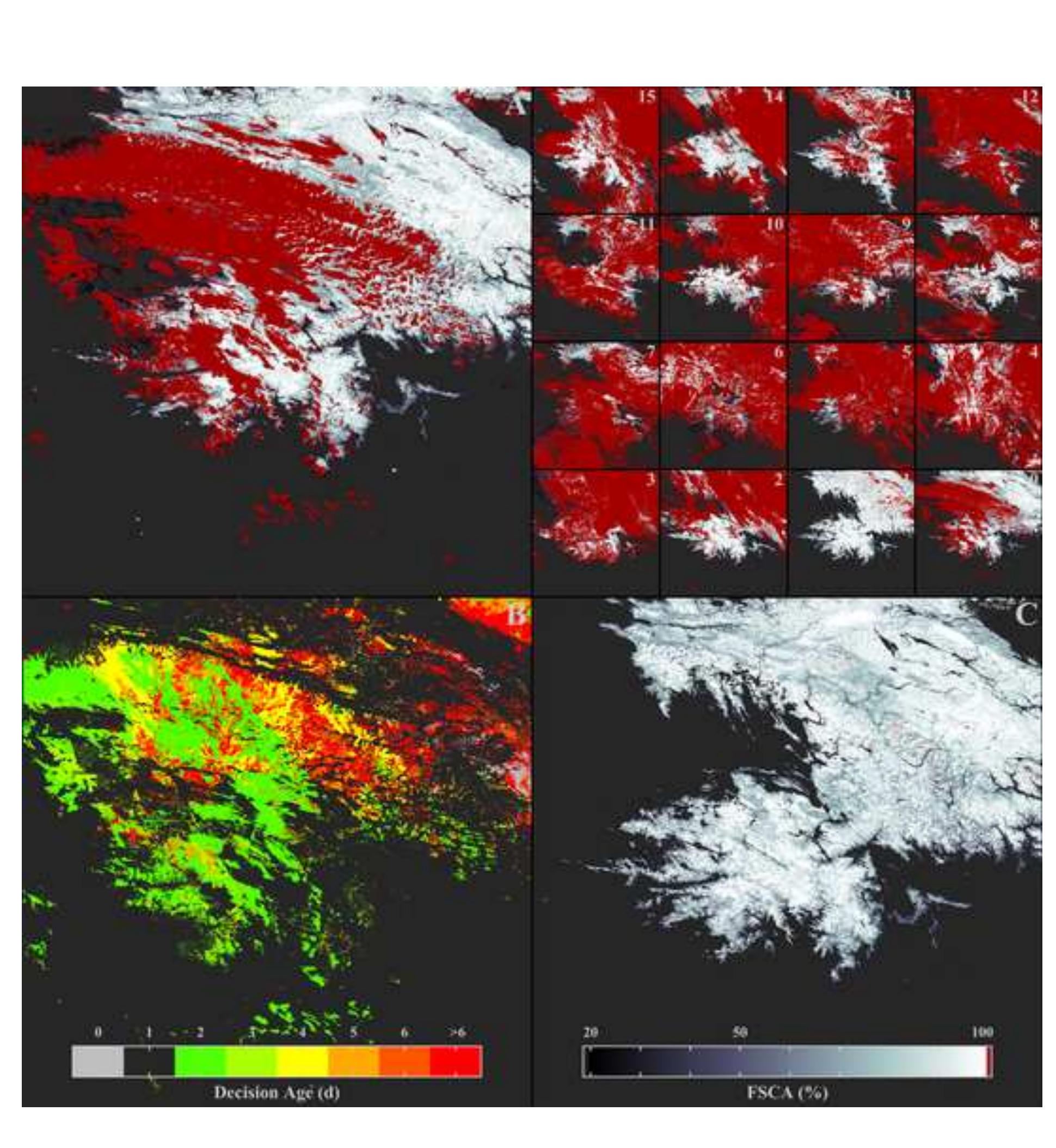

Figure 4

\section{Figure 4}

.

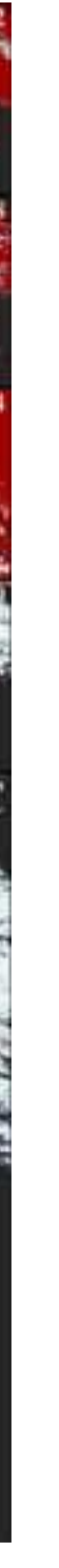

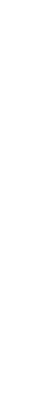

to

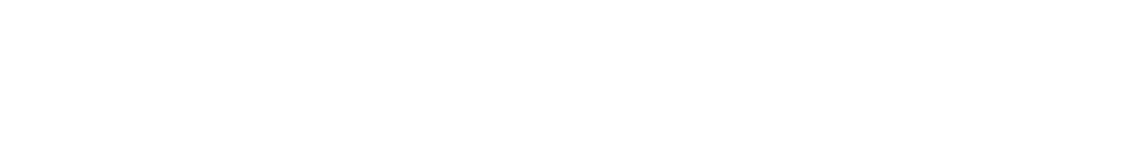




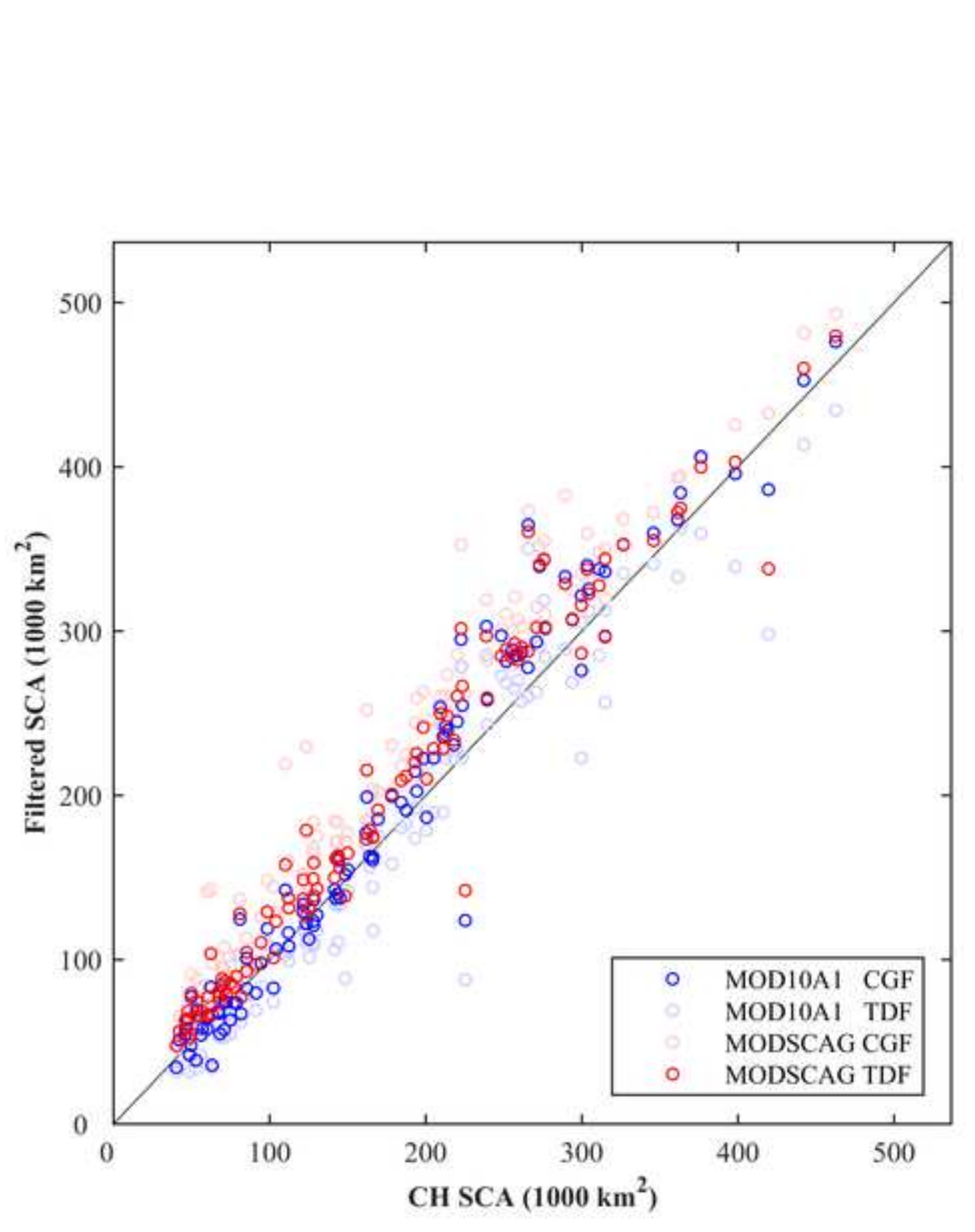

Figure 5

of

\section{5}

(
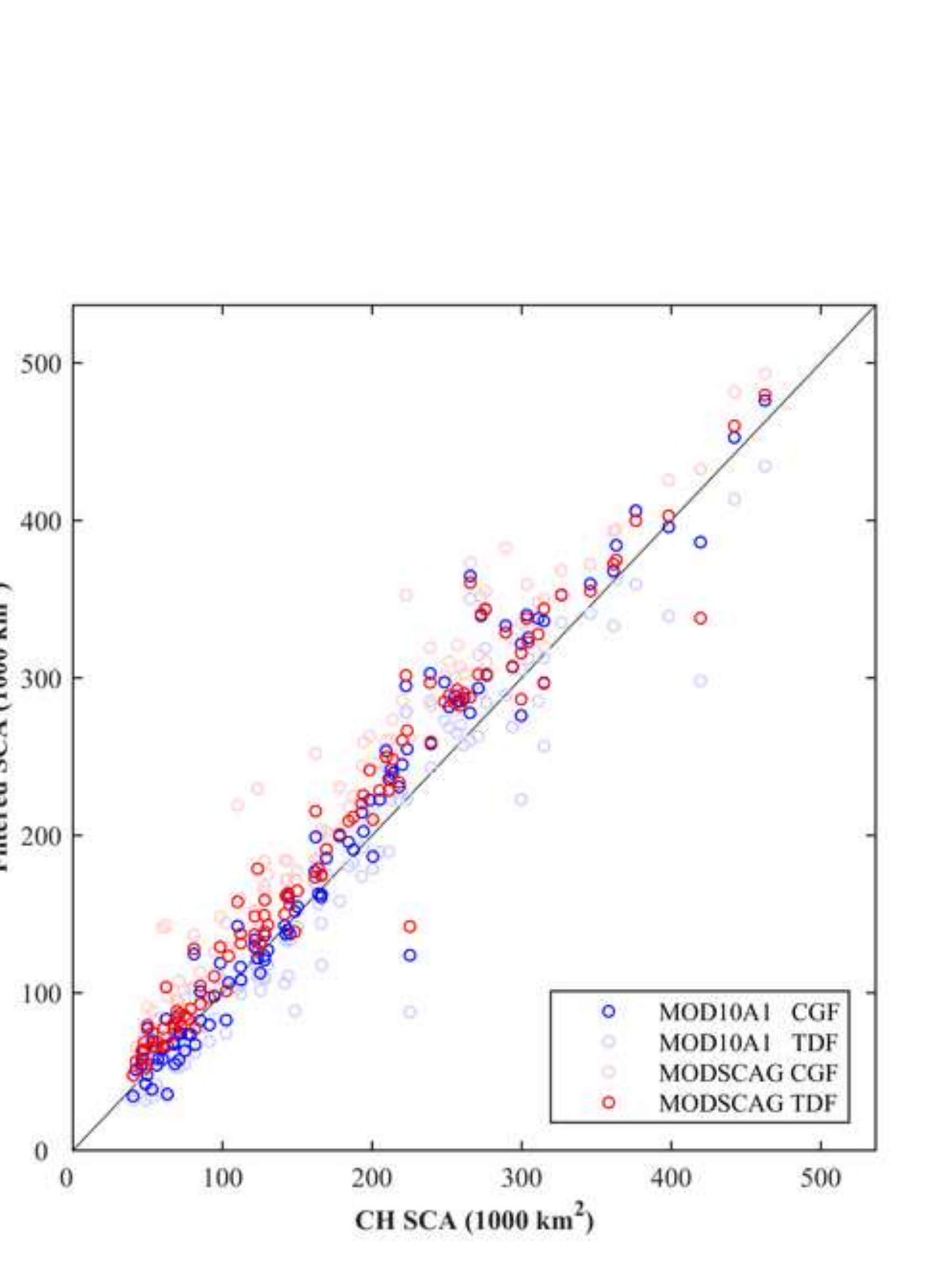

\begin{tabular}{|c}
$\mathbf{p}^{\prime}$ \\
$\mathrm{d}$
\end{tabular}

CHCA $1000 \mathrm{~km})$ 

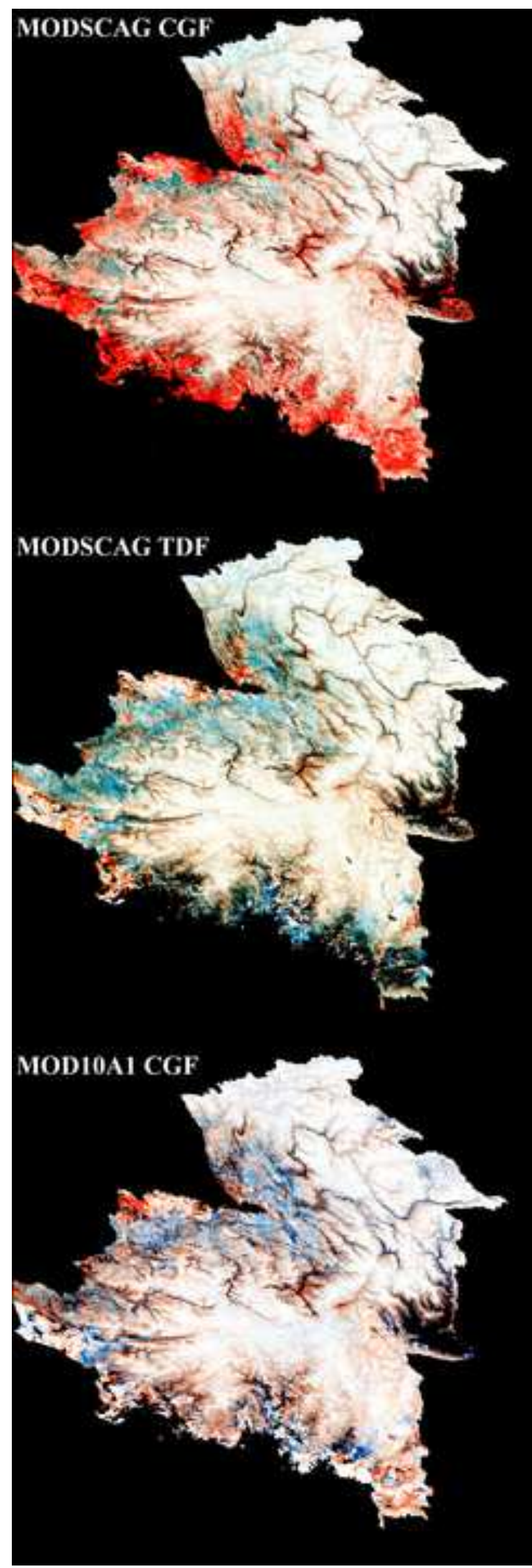